\title{
Meta-analysis on biomechanical properties of meniscus repairs: are devices better than sutures?
}

\author{
Daniel M. Buckland · Patrick Sadoghi - Matthias D. Wimmer • \\ Patrick Vavken · Geert I. Pagenstert • Victor Valderrabano • \\ Claudio Rosso
}

Received: 2 October 2013/Accepted: 19 March 2014/Published online: 20 May 2014

(c) Springer-Verlag Berlin Heidelberg 2014

\begin{abstract}
Purpose Meniscal repair devices have been extensively tested during the past decades as reported in the literature. Reviewing the different meniscal repair devices and sutures with their respective biomechanical properties.

Methods For this meta-analysis, we conducted a systematic online search using PubMed, EMBASE, CCTR, and CINAHL using the search terms Meniscus OR Meniscal AND Biomechanics AND Repair). Load-to-failure $(\mathrm{LtF})$, stiffness, and cyclic outcome measures were extracted independently and in duplicate. The systematic search revealed 841 manuscripts in total. After exclusion of duplicates and irrelevant publications, 41 studies remained
\end{abstract}

Patrick Sadoghi and Dan Buckland have contributed equally to this work.

Electronic supplementary material The online version of this article (doi:10.1007/s00167-014-2966-9) contains supplementary material, which is available to authorized users.

D. M. Buckland · P. Vavken · G. I. Pagenstert · V. Valderrabano · C. Rosso $(\bowtie)$

Orthopaedic Department, University Hospital Basel, Spitalstrasse

21, 4031 Basel, Switzerland

e-mail: Claudio.Rosso@unibas.ch

D. M. Buckland · P. Vavken · G. I. Pagenstert · V. Valderrabano ·

C. Rosso

University of Basel, Basel, Switzerland

P. Sadoghi

Department of Orthopaedic Surgery, Medical University Graz,

Graz, Austria

M. D. Wimmer

Department of Orthopaedic Surgery, University Hospital Bonn,

Bonn, Germany for final analysis. The studies were published in English and German from 1995 to 2013. Due to differing cyclic force protocols, cyclic outcomes had to be excluded.

Results Overall, sutures had a higher LtF [suture: $87.7 \pm 0.3 \mathrm{~N}$ (weighted mean \pm standard error), device: $56.3 \pm 0.1 \mathrm{~N}]$ and stiffness (suture: $8.9 \pm 0.04 \mathrm{~N} / \mathrm{mm}$, device: $8.6 \pm 0.04 \mathrm{~N} / \mathrm{mm}$ ) than devices, both $p<0.05$. In LfT testing, PDS 0 Vertical $(145.0 \pm 8.1 \mathrm{~N})$, OrthoCord $2-0 \quad(143.6 \pm 11.3 \mathrm{~N})$, and Ethibond No 0 Vertical $(133.4 \pm 7.7 \mathrm{~N})$ were the strongest sutures and Meniscal Viper $\quad(140.9 \pm 5.1 \mathrm{~N}), \quad$ MaxFire Vertical $(136.2 \pm 11.3 \mathrm{~N})$, and FasT-Fix Vertical $(115.2 \pm 1.6 \mathrm{~N})$ were the strongest devices. Second-generation devices were significantly stronger and stiffer than first-generation devices $(p<0.001)$.

Clinical relevance Suture repair remains the gold standard with a vertically oriented suture configuration showing superior $\mathrm{LtF}$ values compared to a horizontal configuration. Nevertheless, some meniscal repair devices

\section{D. Wimmer}

University of Bonn, Bonn, Germany

P. Vavken

Sports Medicine Research Laboratory, Department of Orthopedic Surgery, Children's Hospital Boston, Harvard Medical School, Boston, MA, USA

P. Vavken

Harvard Center for Population and Development Studies, Harvard School of Public Health, Boston, MA, USA 
have similar biomechanical properties to suture repairs. Both suture repairs and devices have a place in meniscal restoration.

Level of evidence None, meta-analysis of controlled laboratory studies.

Keywords Meniscus - Repair - Biomechanics · Load-to-failure $\cdot$ Stiffness $\cdot$ Devices

\section{Introduction}

The meniscus is mandatory for shock absorption, load distribution, proprioception, and the knee's overall joint function $[2,12,45]$. It aids in joint stability and protects the cartilage from axial loads, shear stress, and degeneration $[2,12,26,39]$. However, trauma and joint degeneration may lead to meniscal tears and alter the joint's function, leading to acute symptoms and inferior long-term joint conditions [26, 28, 31, 39]. Therefore, the treatment of meniscal tears is one of the most challenging topics in orthopaedic research and sports medicine, and substantial contributions were achieved within the last years [30, 36]. Suturing the tissue of an acute tear in order to maintain its mentioned biomechanical properties has nowadays become the gold standard [3, 41]. Inside-out suture repairs remain the gold standard for meniscal repairs, although this technique is associated with an increased risk of neurovascular injury and is linked to augmented perioperative morbidity [10].

Rigid fixation with initially good clinical outcomes but high mid-term failure rates and damage to cartilage was found with the first-generation all-inside repair devices [4, 18]. Therefore, flexible suture- and anchor-based repair devices were introduced and are nowadays preferred in clinical practice.

It was shown that removal of the meniscus led to increased contact stress with biomechanical wear and permanent deformation of cartilage [29].

However, as the development of devices for meniscus suturing is a growing market, various different applications and techniques have been described in the literature and surgeons might therefore choose from all-inside, insideout, and outside-in sutures as well as different technical devices [12]. As stability of the suture is a crucial outcome parameter, surgeons want to perform best in doing so while using the most adequate and accurate way of suturing. However, scientific literature lacks evidence for a most favourable suture device and technique with respect to biomechanical outcome.

The aim of this study was therefore to evaluate the pooled biomechanical outcome after different techniques and devices for meniscus repair including load-to-failure (LtF) testing as well as stiffness measurements. The hypothesis was that modern devices show at least the same $\mathrm{LtF}$ forces as suture repairs.

\section{Materials and methods}

This study was conducted following the PRISMA statement published by the CONSORT group [33, 34].

Systematic search and strategy

We conducted a systematic review of the literature using the online databases PubMed, MEDLINE, EMBASE, CINAHL (Cumulative Index of Nursing and Allied Health), and CCTR (Cochrane Controlled Trial Register). These electronic databases were searched online for "(Meniscus) OR (Meniscal) AND (biomechanics AND repair)" using these terms as keywords and exploded MeSH terms without restrictions in language or year of publication.

Only studies directly testing meniscus fixation devices in vitro using axial mechanical testing were included. In case of overlapping data, studies were merged as far as possible; completely overlapping studies were excluded. Human and animal studies were included as it has been shown that the tissue differences are minimal [23]. In total, the following studies were included testing human, porcine, and bovine menisci: 18, 28, and 44, respectively. Further exclusion criteria were duplicates and not using a generally accepted testing methodology that was comparable to the existing literature.

\section{Extraction of relevant data}

Eligibility of studies was assessed independently and in duplicate (DB, MDW) and crosschecked to avoid errors. Disagreement was resolved by discussion or, if necessary, with the help of the senior author (CR). The bibliographies of all included studies were reviewed for additional relevant studies. All searches were concluded by 1 January 2013.

The data extracted to categorize the studies were: number of samples, human or type of animal, type of suture or device, and test conditions (cyclic minimum [N], cyclic maximum $[\mathrm{N}]$ ). Outcome data extracted were (when reported): $\mathrm{LtF}$ force $[\mathrm{N}]$, initial/1 cycle displacement [mm], $100,300,500$, and 1,000 cycles displacement $[\mathrm{mm}]$, and stiffness that being defined as the slope of the LtF curve (unit N/mm). The crosshead speed was not considered.

Our search strategy generated 841 studies online and by citation tracking (PubMed: 137, EMBASE: 212, CINAHL: 


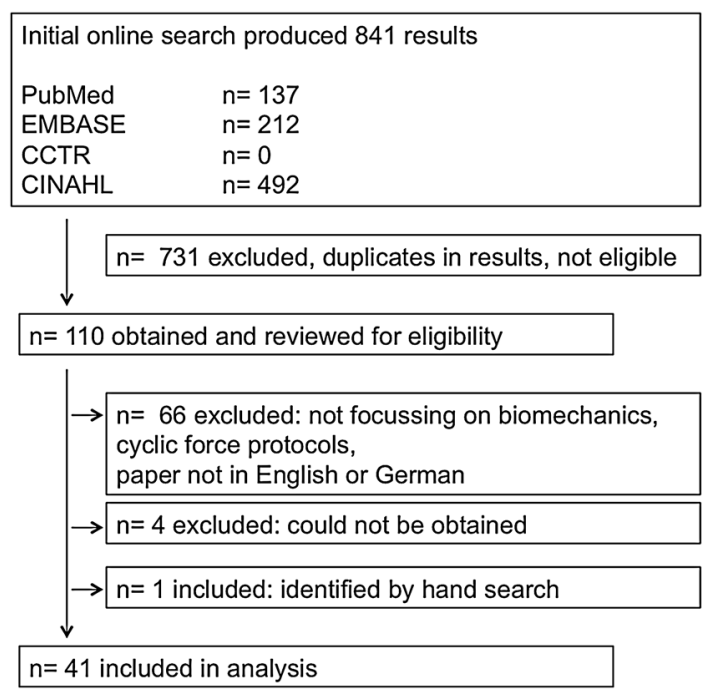

Fig. 1 Study flow chart. This flow chart depicts an overview over the studies in and excluded for this systematic review

492, Cochrane: 0) between 1 January 1995 and 1 January 2013. After exclusion of duplicates, studies not utilizing in vitro uni-axial tension mechanical testing of meniscal repair devices, studies not using comparable loading methods, 41 studies remained for analysis that tested human and animal menisci (Fig. 1). Those 41 studies tested 91 sutures or devices, respectively, out of which $59 \%$ did not explicitly assess cyclic testing. After analysis of these studies, it was evident that cyclic testing was performed in many different manners such as testing at $1 \mathrm{~Hz}$ between 5 and $20 \mathrm{~N}$ [32, 40], 5 and $50 \mathrm{~N}$ [6], and 1 and $11 \mathrm{~N}$ [44], respectively. Due to these inconsistencies, cyclic testing was not included in the final analysis.

Quantitative data synthesis

To pool data, a weighted average and standard error was calculated for each device or suture based on number of samples and standard deviation derived from the studies.

It was attempted wherever possible to combine results from the same sutures and devices reported in different studies. However, due to the discrepancies in how some studies stated the suture used, there may be some results, which could be combined, but this was not done in our analysis. Results reported as a mean and standard deviation were used in meta-analysis. All results below are given as corrected mean [95\% confidence interval (CI) about weighted mean]. First- and second-generation devices were evaluated separately as well as a group (Tables 1, 2). First-generation devices were anchors or screws, while second-generation devices were sutures using an anchor. No IRB approval was needed for this meta-analysis on biomechanical tests.
Statistical analysis

All data were entered into Microsoft Excel (Microsoft, Redmond, WA, USA) and analysed with STATA 10 (StataCorp LP, College Station, TX, USA). The level of significance for pooled estimates was set at $5 \%$.

\section{Results}

Overall results

Overall, sutures had a higher $\operatorname{LtF}$ (mean \pm standard error, SE) suture: $87.7 \pm 0.3 \mathrm{~N}$, device: $56.3 \pm 0.1 \mathrm{~N}$ ), and stiffness (suture: $8.9 \pm 0.04 \mathrm{~N} / \mathrm{mm}$, device: $8.6 \pm 0.04 \mathrm{~N} /$ $\mathrm{mm}$ ) than devices, both with $p<0.05$.

This section mentions the top three sutures and/or devices in the respective section. Further below, for each suture and device LtF (Table 1) and stiffness (Table 2) with the current evidence references are noted. These figures also depict the different first- and second-generation devices. Second-generation devices were significantly stronger and stiffer than first-generation devices $(p<0.001)$. In the next paragraphs, weighted mean $\pm \mathrm{SE}$ are given. Please consult Tables 1 and 2 for CI.

\section{Strongest suture repair (highest load-to-failure)}

Listed are the top three suture repairs with respect to LtF. Data are formatted as weighted mean $\pm \mathrm{SE}$.

1. PDS 0 Vertical with $145.0 \pm 8.1 \mathrm{~N}$ [20]

2. Orthocord 2-0 with $143.6 \pm 11.3 \mathrm{~N}[6,8]$

3. Ethibond No 0 Vertical with $133.4 \pm 7.7 \mathrm{~N}$ [15]

\section{Strongest device (highest load-to-failure)}

1. Meniscal Viper with $140.9 \pm 5.1 \mathrm{~N}[15,20,21]$

2. MaxFire Vertical with $136.2 \pm 11.3 \mathrm{~N}[5,8]$

3. FasT-Fix Vertical with $115.2 \pm 1.6 \mathrm{~N}[5,7,14,15,24$, $47,48]$

Highest stiffness of all repairs stating an SD

1. FiberWire 2-0 Vertical with $28.7 \pm 4.2 \mathrm{~N} / \mathrm{mm}$ [40]

2. Ultrabraid No. 0 with $26.1 \pm 8.1 \mathrm{~N} / \mathrm{mm}$ [40]

3. Ultra FasT-Fix with $25.6 \pm 1.6 \mathrm{~N} / \mathrm{mm}[32,40]$

\section{Strongest repair with respect to orientation of the repair}

Those studies explicitly stating the orientation of the repair were analysed for the load-to-failure. 
Table 1 Load-to-failure summary

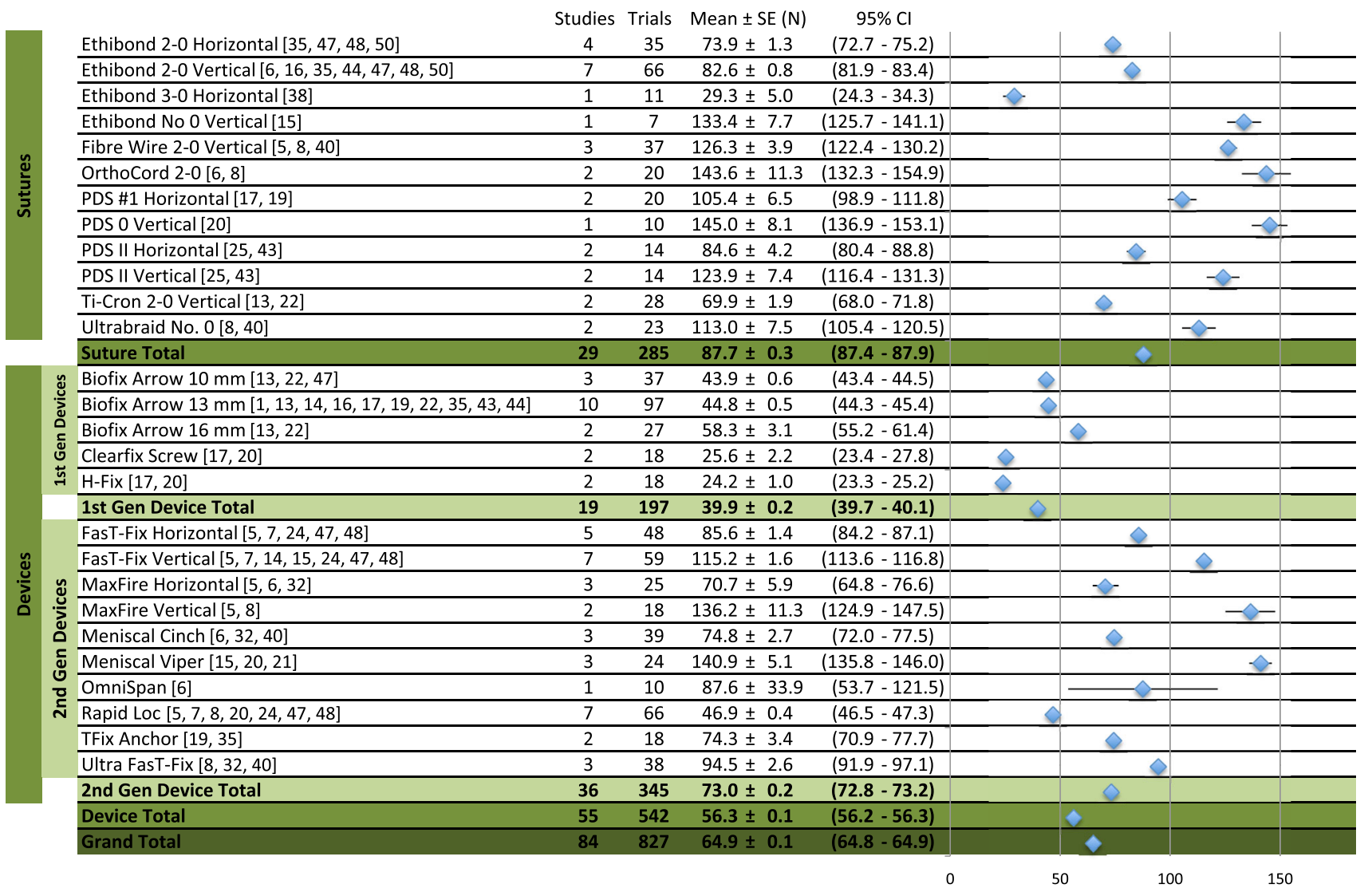

Second-generation devices were significantly stronger compared to the first-generation devices $(p<0.001)$. Dots indicate weighted mean load-tofailure for each device or suture configuration tested. Bars indicate $95 \%$ confidence interval of the mean. Studies = number of studies that tested the device, Trials $=$ number of samples tested. Total study number includes number of studies counted multiple times for each repair method tested

1. Inside-out with $95.5 \pm 2.2 \mathrm{~N}(n=5$ studies $)$

2. $\quad$ Outside-in with $72.7 \pm 1.2 \mathrm{~N}(n=7$ studies $)$

\section{Strongest repair with respect to positioning of the repair}

Those studies explicitly stating the positioning of the repair were analysed for the LtF.

1. Vertical with $97.1 \pm 0.8 \mathrm{~N}$ ( $n=26$ studies $)$

2. Horizontal with $60.2 \pm 0.8 \mathrm{~N}$ ( $n=19$ studies $)$

The following sutures and devices were tested. Please consult Tables 1 and 2 for details.

\section{Sutures}

Resorbable sutures tested were: PDS $\# 1^{\mathrm{TM}}$, PDS $0^{\mathrm{TM}}$, PDS II $^{\mathrm{TM}}$ (Ethicon, Inc., Somerville, NJ, USA)

Non-resorbable sutures tested were: Ethibond ${ }^{\mathrm{TM}}$ No. 0 , Ethibond $^{\mathrm{TM}}$ 2-0 and Ethibond ${ }^{\mathrm{TM}}$ 3-0 (Ethicon, Inc., Somerville, NJ, USA), FibreWire ${ }^{\circledR}$ 2-0 (Arthrex, Inc., Naples,
FL, USA), Orthocord ${ }^{\circledR}$ 2-0 (DePuy Mitek, Raynam, MA, USA), Ti-Cron ${ }^{\mathrm{TM}}$ 2-0 (Covidien, Inc., Mansfield, MA, USA), Ultrabraid ${ }^{\circledR}$ No. 0 (Smith\&Nephew, Andover, MA, USA), Nitinol ${ }^{\circledR}$ 2-0 and Nitinol ${ }^{\circledR}$ 3-0 (Clarimed, Los Gatos, CA, USA), Nylon 2-0 and Nylon 3-0 (Assut Medical SARL, Pully-Lausanne, Switzerland)

Devices tested were: Biofix Arrow ${ }^{\circledR} 10,13$, and $16 \mathrm{~mm}$ (Biofix, Blue Bell, PA, USA), Clearfix ${ }^{\circledR}$ Screw (Innovasive Devices, Marlborough, MA, USA), H-Fix ${ }^{\circledR}$, OmniSpan $^{\mathrm{TM}}$ and Rapid Loc ${ }^{\circledR}$ (DePuy Mitek, Raynam, MA, USA), TFix $^{\circledR}$ Anchor, FasT-Fix ${ }^{\circledR}$ and Ultra FasT-Fix ${ }^{\circledR}$ (Smith\&Nephew, Andover, MA, USA), MaxFire ${ }^{\mathrm{TM}}$ (Biomet, Inc., Warsaw, IN, USA), Meniscal Cinch $^{\circledR}$ and Meniscal Viper $^{\mathrm{TM}}$ (Arthrex, Inc., Naples, FL, USA).

\section{Discussion}

The most important finding was that the vertically oriented repair remains the gold standard in biomechanical testing by virtue of the highest LtF and stiffness. 
Table 2 Stiffness summary

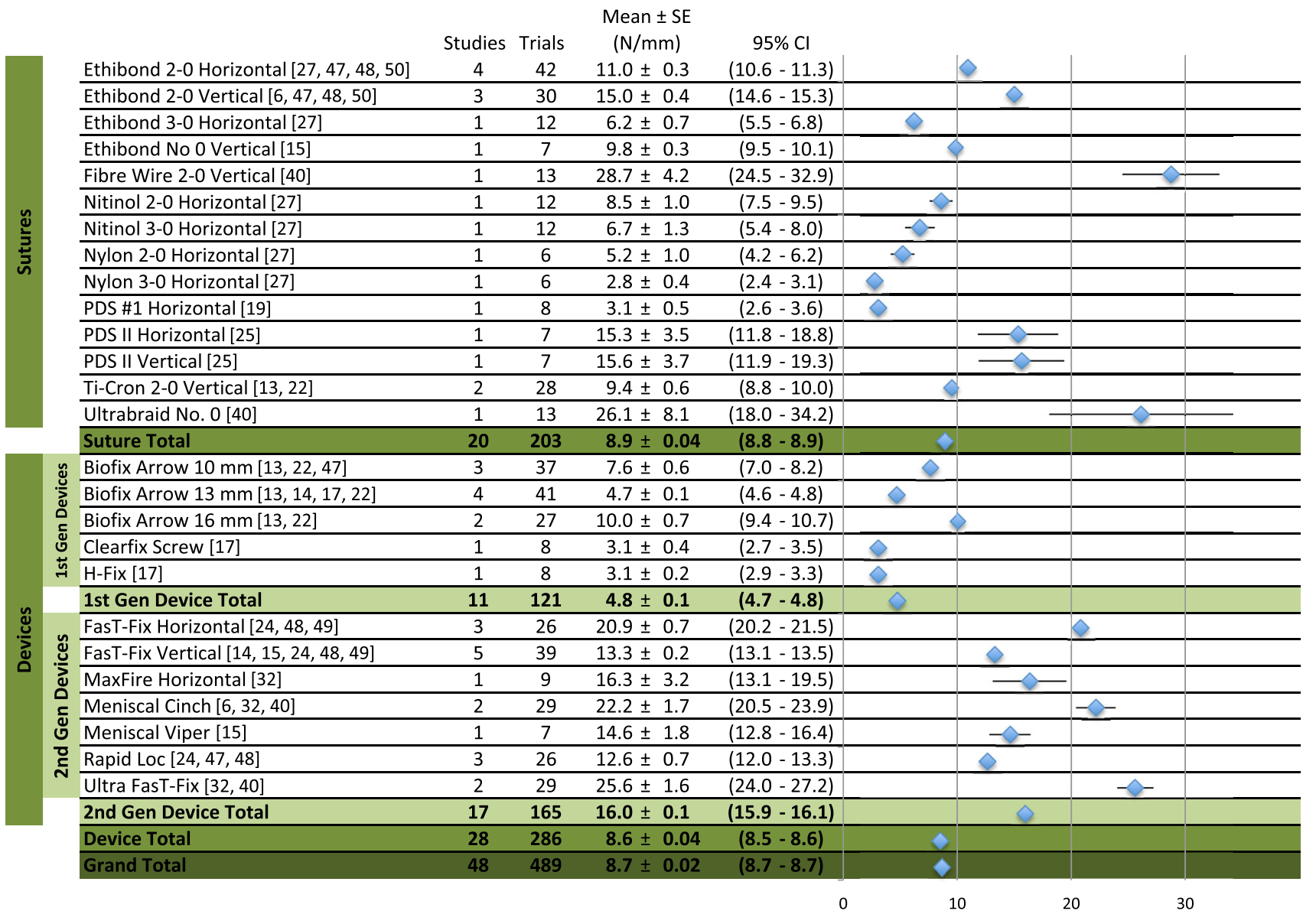

Second-generation devices were significantly stiffer than first-generation devices $(p<0.001)$. Stiffnesses for the Orthocord $2-0$ and the OmniSpan have been reported to be 27.0 and $27.5 \mathrm{~N} / \mathrm{mm}$, respectively, without stating an SD [6]. Thus, those repairs were not taken into the calculations. Dots indicate weighted mean stiffness for each device or suture configuration tested. Bars indicate $95 \%$ confidence interval of the mean. Studies = number of studies that tested device, Trials = number of samples tested. Total study number includes number of studies counted multiple times for each repair method tested

As shown by McCann et al. [29], removal of the meniscus leads to cartilage degeneration with its final stage: osteoarthritis.

Although meniscus repair has evolved during the last decades, meniscal repair devices are still compared to the gold standard of inside-out repairs. Meniscal repair devices are mostly used for posterior horn tears as well as intermediate part tears while suture techniques are preferred for anterior horn tears or detachments. Still, for the anterior horn, suture techniques are commonly used due to the ease in this area. To date, 841 studies have been found that report on meniscus repairs and its biomechanical properties. This impressive amount of studies makes it difficult for orthopaedic surgeons to choose the right repair device. With this comprehensive meta-analysis, we are trying to help in this difficult decisionmaking process.
Summary of evidence

In this meta-analysis, we tried to include all relevant biomechanical evaluations of meniscal repair devices since the year 1995 up to 1 January 2013.

Higher LtF measurements are considered to be superior in the evaluation of devices and sutures for meniscal repair. Nevertheless, a threshold has never been established and there is still dispute on the amount of in vivo loads to the human meniscus $[11,37]$. This makes the final evaluation of the different $\mathrm{LtF}$ measurements difficult to interpret.

Another value that has been frequently reported is stiffness. Again, there has been no consensus as to the appropriate threshold value. Regarding biomechanical characteristics, the stiffness of a construct should not be too high (rigid) in order not to have too high sheer forces between the repair and the construct, but the agreement as 
to what too a high a value might does not exist. In our belief, stiffness is not as important as LtF testing. Stiff materials are considered to be stronger than elastic materials. Nevertheless, stiff materials might more easily cut through soft tissue due to the difference in stiffness. Cyclic testing, which has not been performed in $59 \%$ of the studies analysed, seems to be even more important. We strongly believe that this is the best way to measure the quality of a repair. As reported, two biomechanical factors influence the quality of a repair: (1) initial gapping between the torn meniscus parts at cycle 1 and (2) increase in gapping during cyclic loading [40]. If initial gapping is too high, there is too little contact area between the meniscus parts. On the other hand, if the increase in gapping during cyclic loading is too excessive, initial contact area might be lost. In a worst-case scenario, a 30-year-old man in a walking intensive profession (postman) takes about 12,000 steps a day [42]. Excessive cyclic gapping might thus impair postoperative healing. This makes biomechanical testing, although uni-axial, important.

Although healing rates of up to $83 \%$ with repeat arthroscopies have been reported $[9,46]$, we do not know if a meniscus completely heals after having undergone repairs. The most commonly used sutures are non-resorbable sutures. It is not known if these are used due to their higher LtF rates or in order to keep the meniscus stable over an extended time span. Second-generation meniscal repair devices show similar biomechanical properties compared to inside-out suture repairs and could thus be used in daily clinical work [6]. Our study has potential shortcomings. Like any meta-analyses, the validity of our findings depends on the validity of the primary studies. Another shortcoming is the heterogeneity of the included primary studies. This is the reason why we did not preform any statistical analysis but to state the large amount of biomechanical outcomes. Although we tried to include comparable studies, several donor species and slight variation in testing methods were included, which might significantly contribute to the error in comparing biomechanical testing [23]. As stated in practically all biomechanical meniscus studies, the uni-axial loading recreates a worstcase scenario and does not reproduce sheer forces. We did not consider different crossheads speeds. Most studies use speeds at $3.15 \mathrm{~mm} / \mathrm{s}$, but this speed was not consistently used and might thus alter stiffness and LtF values.

\section{Conclusions}

Suture repairs remain the gold standard in terms of biomechanical characteristics. A vertically oriented repair seems to be superior to the horizontal orientation based on higher LtF and stiffness values. Meniscal repair devices are nevertheless evolving with respect to $\mathrm{LtF}$ testing. Cyclic testing should always be performed when evaluating the biomechanical properties of meniscal repairs.

Acknowledgments We thank all the authors whose studies we analysed for their contribution. Dr. Buckland thanks the Whitaker International Foundation for fellowship support.

\section{References}

1. Albrecht-Olsen P, Lind T, Kristensen G, Falkenberg B (1997) Failure strength of a new meniscus arrow repair technique: biomechanical comparison with horizontal suture. Arthroscopy 13(2): $183-187$

2. Allen CR, Wong EK, Livesay GA, Sakane M, Fu FH, Woo SL (2000) Importance of the medial meniscus in the anterior cruciate ligament-deficient knee. J Orthop Res 18(1):109-115

3. Allen PR, Denham RA, Swan AV (1984) Late degenerative changes after meniscectomy: factors affecting the knee after operation. J Bone Jt Surg Br 66(5):666-671

4. Anderson K, Marx RG, Hannafin J, Warren RF (2000) Chondral injury following meniscal repair with a biodegradable implant. Arthroscopy 16(7):749-753

5. Aros BC, Pedroza A, Vasileff WK, Litsky AS, Flanigan DC (2010) Mechanical comparison of meniscal repair devices with mattress suture devices in vitro. Knee Surg Sports Traumatol Arthrosc 18(11):1594-1598

6. Barber FA, Ma Herbert, Bava ED, Drew OR (2012) Biomechanical testing of suture-based meniscal repair devices containing ultrahigh-molecular-weight polyethylene suture: update 2011. Arthroscopy 28(6):827-834

7. Barber FA, Ma Herbert, Richards DP (2004) Load to failure testing of new meniscal repair devices. Arthroscopy 20(1):45-50

8. Barber FA, Herbert MA, Schroeder FA, Aziz-Jacobo J, Sutker MJ (2009) Biomechanical testing of new meniscal repair techniques containing ultra high-molecular weight polyethylene suture. Arthroscopy 25(9):959-967

9. Barber FA, Schroeder FA, Oro FB, Beavis RC (2008) FasT-Fix meniscal repair: mid-term results. Arthroscopy 24(12):13421348

10. Barrett GR, Richardson K, Ruff CG, Jones A (1997) The effect of suture type on meniscus repair: a clinical analysis. Am J Knee Surg 10(1):2-9

11. Becker R, Brettschneider O, Grobel KH, von Versen R, Starke C (2006) Distraction forces on repaired bucket-handle lesions in the medial meniscus. Am J Sports Med 34(12):1941-1947

12. Bedi A, Kelly N, Baad M, Fox AJ, Ma Y, Warren RF, Maher SA (2012) Dynamic contact mechanics of radial tears of the lateral meniscus: implications for treatment. Arthroscopy 28(3):372-381

13. Boenisch UW, Faber KJ, Ciarelli M, Steadman JR, Arnoczky SP (1999) Pull-out strength and stiffness of meniscal repair using absorbable arrows or Ti-Cron vertical and horizontal loop sutures. Am J Sports Med 27(5):626-631

14. Borden P, Nyland J, Caborn DNM, Pienkowski D (2003) Biomechanical comparison of the FasT-Fix meniscal repair suture system with vertical mattress sutures and meniscus arrows. Am J Sports Med 31(3):374-378

15. Chang HC, Nyland J, Caborn DNM, Burden R (2005) Biomechanical evaluation of meniscal repair systems: a comparison of the Meniscal Viper repair system, the vertical mattress FasT-Fix Device, and vertical mattress ethibond sutures. Am J Sports Med 33(12):1846-1852 
16. Dervin GF, Downing KJ, Keene GC, McBride DG (1997) Failure strengths of suture versus biodegradable arrow for meniscal repair: an in vitro study. Arthroscopy 13(3):296-300

17. Dürselen L, Schneider J, Galler M, Claes LE, Bauer G (2003) Cyclic joint loading can affect the initial stability of meniscal fixation implants. Clin Biomech 18(1):44-49

18. Ellermann A, Siebold R, Buelow JU, Sobau C (2002) Clinical evaluation of meniscus repair with a bioabsorbable arrow: a 2- to 3-year follow-up study. Knee Surg Sports Traumatol Arthrosc 10(5):289-293

19. Fisher SR, Markel DC, Koman JD, Atkinson TS (2002) Pull-out and shear failure strengths of arthroscopic meniscal repair systems. Knee Surg Sports Traumatol Arthrosc 10(5):294-299

20. Gunes T, Bostan B, Erdem M, Asci M, Sen C, Kelestemur MH (2009) Biomechanical evaluation of arthroscopic all-inside meniscus repairs. Knee Surg Sports Traumatol Arthrosc 17(11):1347-1353

21. Gunes T, Bostan B, Erdem M, Asci M, Sen C, Kelestemur MH (2011) The "butterfly" suture technique for meniscal repair. Arch Orthop Trauma Surg 131(3):331-333

22. Jani MM (2004) Mechanical properties of bioabsorbable meniscal arrows as a function of tear location: an ex vivo experimental study. Am J Sports Med 32(3):666-674

23. Joshi MD, Suh JK, Marui T, Woo SL (1995) Interspecies variation of compressive biomechanical properties of the meniscus. J Biomed Mater Res 29(7):823-828

24. Kocabey Y, Chang HC, Brand JC, Nawab A, Nyland J, Caborn DNM (2006) A biomechanical comparison of the FasT-Fix meniscal repair suture system and the RapidLoc device in cadaver meniscus. Arthroscopy 22(4):406-413

25. Kocabey Y, Taser O, Nyland J, Doral MN, Demirhan M, Caborn DNM, Sarban S (2006) Pullout strength of meniscal repair after cyclic loading: comparison of vertical, horizontal, and oblique suture techniques. Knee Surg Sports Traumatol Arthrosc 14(10):998-1003

26. Krause WR, Pope MH, Johnson RJ, Wilder DG (1976) Mechanical changes in the knee after meniscectomy. J Bone Jt Surg Am 58(5):599-604

27. Aa Lamprakis, Fortis AP, Kostopoulos V, Vlasis K (2009) Biomechanical testing of a shape memory alloy suture in a meniscal suture model. Arthroscopy 25(6):632-638

28. Markolf KL, Mensch JS, Amstutz HC (1976) Stiffness and laxity of the knee-the contributions of the supporting structures: a quantitative in vitro study. J Bone Jt Surg Am 58(5):583-594

29. McCann L, Ingham E, Jin Z, Fisher J (2009) Influence of the meniscus on friction and degradation of cartilage in the natural knee joint. Osteoarthr Cartil 17(8):995-1000

30. McDermott ID, Amis AA (2006) The consequences of meniscectomy. J Bone Jt Surg Br 88(12):1549-1556

31. Meakin JR, Shrive NG, Frank CB, Hart DA (2003) Finite element analysis of the meniscus: the influence of geometry and material properties on its behaviour. Knee 10(1):33-41

32. Mehta VM, Terry MA (2009) Cyclic testing of 3 all-inside meniscal repair devices: a biomechanical analysis. Am J Sports Med 37(12):2435-2439

33. Moher D, Cook DJ, Eastwood S, Olkin I, Rennie D, Stroup DF (1999) Improving the quality of reports of meta-analyses of randomised controlled trials: the QUOROM statement. Quality of reporting of meta-analyses. Lancet 354(9193):1896-1900
34. Moher D, Liberati A, Tetzlaff J, Altman DG (2009) Preferred reporting items for systematic reviews and meta-analyses: the PRISMA statement. J Clin Epidemiol 62(10):1006-1012

35. Rankin CC, Lintner DM, Noble PC, Paravic V, Greer E (2002) A biomechanical analysis of meniscal repair techniques. Am J Sports Med 30(4):492-497

36. Renstrom P, Johnson RJ (1990) Anatomy and biomechanics of the menisci. Clin Sports Med 9(3):523-538

37. Richards DP, Barber FA, Herbert MA (2005) Compressive loads in longitudinal lateral meniscus tears: a biomechanical study in porcine knees. Arthroscopy 21(12):1452-1456

38. Rimmer MG, Nawana NS, Keene GC, Pearcy MJ (1995) Failure strengths of different meniscal suturing techniques. Arthroscopy 11(2): $146-150$

39. Rodkey WG (2000) Basic biology of the meniscus and response to injury. Instr Course Lect 49:189-193

40. Rosso C, Kovtun K, Dow W, McKenzie B, Nazarian A, Deangelis JP, Ramappa AJ (2011) Comparison of all-inside meniscal repair devices with matched inside-out suture repair. Am J Sports Med 39(12):2634-2639

41. Seedhom BB, Dowson D, Wright V (1974) Proceedings: functions of the menisci. A preliminary study. Ann Rheum Dis 33(1): 111

42. Sequeira MM, Rickenbach M, Wietlisbach V, Tullen B, Schutz Y (1995) Physical activity assessment using a pedometer and its comparison with a questionnaire in a large population survey. Am J Epidemiol 142(9):989-999

43. Song EK, Lee KB (1999) Biomechanical test comparing the load to failure of the biodegradable meniscus arrow versus meniscal suture. Arthroscopy 15(7):726-732

44. Staerke C, Bochwitz C, Groebel K-H, Unterhauser F, Becker R (2004) The effect of meniscus compression on the biomechanical properties of repaired meniscal lesions. Winner of the AGADonJoy Award 2003. Arch Orthop Trauma Surg 124(4):221-225

45. Voloshin AS, Wosk J (1983) Shock absorption of meniscectomized and painful knees: a comparative in vivo study. J Biomed Eng 5(2):157-161

46. Xu C, Zhao J (2013) A meta-analysis comparing meniscal repair with meniscectomy in the treatment of meniscal tears: the more meniscus, the better outcome? Knee Surg Sports Traumatol Arthrosc [Epub ahead of print]

47. Zantop T (2004) Initial fixation strength of flexible all-inside meniscus suture anchors in comparison to conventional suture technique and rigid anchors: biomechanical evaluation of new meniscus refixation systems. Am J Sports Med 32(4):863-869

48. Zantop T (2005) Cyclic testing of flexible all-inside meniscus suture anchors: biomechanical analysis. Am J Sports Med 33(3):388-394

49. Zantop T, Eggers AK, Musahl V, Weimann A, Hassenpflug J, Petersen W (2004) A new rigid biodegradable anchor for meniscus refixation: biomechanical evaluation. Knee Surg Sports Traumatol Arthrosc 12(4):317-324

50. Zantop T, Temmig K, Weimann A, Eggers AK, Raschke MJ, Petersen W (2006) Elongation and structural properties of meniscal repair using suture techniques in distraction and shear force scenarios: biomechanical evaluation using a cyclic loading protocol. Am J Sports Med 34(5):799-805 